

\title{
TOURAINE, A. (2018): DÉFENSE DE LA MODERNITÉ. 384 PP. PARIS: SEUIL
}

\author{
Prof. Eguzki Urteaga ${ }^{1}$ \\ Universidad del País Vasco, España
}

Alain Touraine acaba de publicar su último libro titulado Défense de la modernité (Defensa de modernidad) en la editorial Seuil. Es preciso recordar que, tras crear el Laboratorio de Sociología Industrial, que se convirtió en el Centro de los Movimientos Sociales de la Escuela Práctica de Altos Estudios en Ciencias Sociales, este sociólogo galo fundó y dirigió el Centro de Análisis y de Intervenciones Sociológicas. Tras iniciar su carrera profesional como investigador del CNRS, se convirtió en Director de Estudios en la Escuela de Altos Estudios en Ciencias Sociales de París y fue igualmente catedrático de sociología durante un breve periodo en la Facultad de Letras de la Universidad de París X-Nanterre. Su labor es internacionalmente reconocida, convirtiéndose en una de las principales figuras de la sociología a nivel mundial. Prueba de ello es que ha sido nombrado doctor honoris causa por numerosas universidades francesas y extranjeras, entre las cuales podemos citar las Universidades de Ginebra, Montreal, Lovaina, Bolonia, Nueva York, México, Santiago de Chile, Colombia, Córdoba, La Paz, Perú, etc. Autor de una abundante bibliografía, su obra aspira a crear una sociología de la acción que se ha convertido progresivamente en una sociología del sujeto y del proceso de subjetivación, al considerar que la experiencia moderna debe reconstruirse en torno a esta figura.

Défense de la modernité se inscribe plenamente en esta óptica. En la introducción de esta obra, el autor subraya que su "adversario principal, el que busca más constantemente (...) [reducirlo] al silencio como sociólogo, es la idea de que nuestras situaciones y acciones están dominadas por las leyes de la economía" (p. 11). De hecho, Touraine ha luchado toda su vida intelectual contra el pensamiento dominante, tanto de derechas como de izquierdas, que se apoya en "el determinismo económico" (p. 11). En ese sentido, desea liberar la mente de todas las formas de determinismo a las que se han querido encadenar las conductas humanas (p. 11). "Es porque las prácticas creadoras de historia descartan la idea de naturaleza humana, creada por un Dios todo-poderoso, que esta visión de las sociedades modernas nos libera de las ilusiones religiosas; es porque los conflictos sociales centrales, los

\footnotetext{
${ }^{1}$ Departamento de Sociología y Trabajo Social. Facultad de Relaciones Laborales y Trabajo Social.
} E-mail: eguzki.urteaga@ehu.eus 
que oponen los posesores a los dependientes, se transforman con las formas de la civilización material (...) que debemos liberarnos de las ilusiones económicas y políticas; por último, es porque la modernidad es ante todo historicidad y cambio que debemos liberarnos también de la ilusión de que nuestras conductas deben conformarse a las exigencias funcionales (...) de la vida social” (p.14).

La esfera de la acción social, es decir la "de la libertad, de la creatividad y de la modernidad, está definida por la interdependencia completa de (...) tres componentes: la creación y transformación de una civilización material; la representación asociada a esta práctica de una conciencia de la creatividad en una sociedad definida por (...) su historicidad; y, por último, la conflictividad central" (pp. 14-15). Esto implica proceder a una profunda refundación "de nuestro conocimiento de la vida social" (p. 15).

De hecho, la sociedad hipermoderna en la que vivimos, nos dice el autor, se caracteriza por "su plena y directa conciencia de sí misma como [entidad] autotransformadora y creadora de sí misma” (p. 16). Es la razón la cual, "el objetivo principal y directo es el de [fomentar] la creatividad", lo que otorga un lugar central a la educación (p.16). En esta nueva sociedad, “el poder no es solamente político y económico (...), sino que es también cultural, puesto que las comunicaciones [difunden] unas informaciones que transforman las conductas, las actitudes y las representaciones, los proyectos y los [estilos] de vida" (pp. 16-17). Esto obliga "los movimientos [sociales] y las fuerzas políticas, que pretenden resistir a los poderes totales, que se multiplican [por todas partes], a actuar a un nivel global, defendiendo, no solamente unos derechos particulares (...), sino [también] el sujeto humano [como tal] en sus derechos fundamentales" (p. 17).

Así, la conciencia social se convierte en el lugar central de los conflictos. Para Touraine, "el siglo XXI será el del enfrentamiento entre la subjetivación y la desubjetivación" (p. 17). Por lo tanto, "en lugar de prohibir o de debilitar los esfuerzos de subjetivación, es decir el respeto de los derechos humanos fundamentales, debemos combatir las políticas de desubjetivación, es decir el rechazo de defender la libertad, la igualdad y la dignidad de todos los seres humanos" (p. 18).

Dentro de la primera parte, titulada "Modernidad y subjetivación", el autor dedica el primer capítulo a las "prácticas e interpretaciones de la modernidad" (p. 33). Constata que las sociedades modernas "se definen por una acción de creación, de transformación, pero también de destrucción de sí mismas. Se trata, por lo tanto, (...) de definirlas por su historicidad, su capacidad de producir historia. Comprenderlas significa, ante todo, analizar lo que las convierte en creadoras de su historia" ( $p$. 33). En otras palabras, define la modernidad "por la voluntad y la capacidad de ciertas sociedades para crearse, transformarse y (...) destruirse, a la vez, para lo mejor y para lo peor" (p.34). En efecto, las sociedades modernas adquieren una 
conciencia creciente de no ser "unas criaturas de un orden divino o natural, sino unas creadoras de sí mismas" (p. 34). No solamente los entornos naturales son transformados con la ayuda de dispositivos técnicos, sino que, además, "la acción humana es cada vez más reflexiva y [adquiere] un conocimiento de sí misma como creadora y transformadora, modificando unos entornos técnicos ya establecidos" (p. 34). Bajo el efecto de los avances científicos y de las innovaciones técnicas, $\mathrm{y}$, más aún, como consecuencia de las catástrofes del siglo XX, estas sociedades "han adquirido (...) la conciencia de su capacidad ilimitada de destrucción [y] de transformación" (pp. 35-36).

A su vez, uno de los elementos fundamentales de las sociedades modernas es su conflictividad, ya que, "para transformarse, una sociedad debe acumular unos recursos e invertirlos en unas actividades productivas. La distancia se incrementa, sin cesar, entre los que tienen la capacidad de invertir y los que están obligados a ser dependientes de los grupos dominantes" (p. 38). Los dominantes extienden de manera creciente su dominación, añadiendo a la dominación de los cuerpos, de las leyes y del trabajo, "la dominación de la subjetividad, de las actitudes y de las opiniones, del espacio de la decisión y de las elecciones de vida" (p. 38). Para el autor, la solución consiste en distinguir claramente "la liberación del ser humano como sujeto humano creador" y la del individuo consumidor y, por lo tanto, "dominado por los dueños de la publicidad, de la creación y de la distribución de los recursos y de la subjetividad" (p. 38).

La modernidad se convierte en hipermodernidad cuando los modernos son conscientes de serlo y "están animados por la conciencia de (...) su dignidad como sujetos humanos, creadores libres e iguales" (p. 39). La afirmación de sí mismo "como sujeto libre y respetado es la forma más elevada de esta conciencia personal y colectiva, de ser creador y transformador de sí mismo y del mundo" (p. 40). Estas sociedades, "porque se definen en términos de creación y de cambio, y, por lo tanto, de desdoblamiento de los individuos y de las instituciones entre el universo de la creación y el universo del funcionamiento, llevan en ellas la separación de un mundo de derechos y de un mundo de deberes" (p. 43).

La interdependencia entre los tres componentes principales de las sociedades modernas, a saber "una civilización material, una interpretación cultural de la modernidad creadora $[y]$ una conflictividad social que opone dominantes y dominados", se basa en la naturaleza histórica de los tres componentes (p. 44). Esta naturaleza histórica es evidente en lo que concierne la civilización material, sabiendo que, "en cada civilización, el elemento dominante de la vida social es diferente" (p. 44). Además, "no hay una fractura imposible de franquear entre la civilización material y la interpretación cultural de la creatividad" (p. 45). En efecto, las sociedades son, ante todo, aquellas que tienen "la conciencia de querer ser" (p. 
45). "La manera en la cual la representación de la creatividad toma unas formas institucionales o culturales diferentes según las civilizaciones (...), constituye [la] interpretación" (p. 45). Y, el tercer componente básico de la vida de estas sociedades es "la conflictividad social entre dominantes y dominados" (p. 46).

La definición de los tres componentes básicos de la modernidad carecería de sentido "si no se reconociera la autonomía y, por lo tanto, la separación de estas tres lógicas [así como] su interdependencia" (p. 48). De hecho, "no hay modernidad sin reconocimiento del orden de la producción, es decir sin respeto de la razón instrumental y, más fundamentalmente, de la razón científica” (p. 48). Asimismo, "una sociedad que no reconoce la necesidad de la inversión en la producción y en la formación de los actores humanos no [podría] ser [considerada como] moderna" (p. 48). Pero, indica Touraine, "es la creación de una interpretación de la creatividad humana que constituye el eje principal [y] específico de la modernidad, y es, por lo tanto, su libertad y su defensa activa que constituye la condición principal de la existencia de una sociedad moderna" (p. 49).

Para el sociólogo galo, es preciso conceder "una gran importancia a la separación de los tres elementos de la modernidad" (p. 50). De hecho, no hay modernidad sin separación de poderes y sin un poder limitado. A su vez, la modernidad es imposible "sin respeto del conocimiento, de la investigación y de la innovación, [y] sin respeto de los derechos humanos fundamentales" (p. 50). Por último, "los conflictos sociales, ante todo entre los grupos dominantes y las categorías dependientes, son indispensables a la modernidad" (p. 50). En general, "se da el sentido más amplio y más elevado a las tres dimensiones de la acción social cuando se [comprenden] en su interdependencia y, por lo tanto, en su unidad" (p. 51).

Según Touraine, el incremento global de la capacidad de acción de las sociedades sobre sí mismas justifica la noción de sociedad hipermoderna (p.53). Esta idea debe ser completada por dos observaciones. Por una parte, "la modernidad puede desembocar en unas catástrofes [como consecuencia] de la intervención humana" (p. 53). Por otra parte, "el análisis de las sociedades modernas debe transcender todas las consideraciones morales que pretenden dar la prioridad a la integración social sobre la capacidad y la voluntad de cambio" (p. 53).

A ese respecto, conviene indicar que la idea de sistema social, que ha servido durante un largo periodo a unificar las tres dimensiones, ha estallado literalmente. "Vivimos en un mundo globalizado que rechaza el llamamiento al sujeto" que se ha desvinculado, a su vez, de la idea de progreso económico y social" (p. 56). Por lo cual, es preciso renunciar a la idea de sistema integrado que representa la sociedad, para privilegiar las nociones de actor y de sujeto "que pueden dar sentido a la experiencia hipermoderna" (p. 56). De hecho, para el autor, "la modernidad 
está [parcialmente] vinculada a la conducta del actor, y el estudio de las etapas de la modernidad es la historia de la emergencia del ser humano como sujeto" (p. 56). Por lo tanto, se interesa, ante todo, por "el estudio de las prácticas y, sobre todo, por el trabajo [como] factor principal del cambio histórico" (p.57). Estas prácticas son la base de "una representación del actor por él mismo como creador" (p. 57). En otras palabras, "el actor social es el sujeto en acción, que busca introducir su exigencia de subjetivación en una situación social dada" (p. 57).

La modernidad combina "las tres dimensiones, económica, cultural y sociopolítica", mientras que "el orden se apoya en un principio único de integración" (p. 59). "Contra la unidad represiva del orden estatal, religioso o comunitario, la modernidad integra y respeta los intereses y los deseos individuales, al tiempo que la universalización de los derechos humanos y la búsqueda de la integración social" (p. 60). Pero, nos advierte el pensador galo, es necesario no defender "una imagen demasiado conquistadora, e incluso triunfadora, de la modernidad", ya que lleva en sí misma "una fragilidad" (p. 60). "La modernidad es un modo de existencia que conviene a todos aquellos que no se identifican todavía e incluso que rechazan completamente identificarse a un país, a una profesión, a un entorno o a una opinión” (pp. 60-61).

La modernidad preocupa, "porque es lo contrario del orden, de lo absoluto y de lo ideal (...), al tiempo que estimula y pone en movimiento. Suscita la inquietud y la insatisfacción" (p. 61). Porque "pone en relación el individualismo del deseo y el universalismo de los derechos, el trabajo [y] la conciencia de sí mismo y la modernidad misma, excita la imaginación, pero agrava también los conflictos. Se opone, ante todo, a la idea de una sociedad de orden y de identidad" (p. 61). El peor enemigo de la modernidad es la idea de progreso al generar expectativas excesivas, ya que ninguna sociedad es capaz de "garantizar a todos que su experiencia superará siempre sus expectativas. Es el pensamiento constante del fracaso, del retroceso e incluso de la inamovilidad vivida y comparada con los avances de otros actores (...) que provoca la insatisfacción, la decepción, el desánimo [y] el rechazo” (p. 61).

La sociedad hipermoderna implica la movilización de "movimientos sociales totales, (...) a la vez, éticos y democráticos, para luchar contra poderes ellos mismos convertidos en totales" (p. 65). En ese nuevo tipo de sociedad, indica Touraine, es preciso "afirmar el carácter global del análisis sociológico y rechazar firmemente la generalidad de las explicaciones económicas" (p. 65). En la nueva sociedad, las personas están preocupadas por "el respeto de la dignidad y, en particular, de los derechos culturales de cada uno" (p.65). Esta sociedad naciente, "fundada en las nuevas tecnologías de [la información y de la] comunicación y en la globalización de la economía, en lugar de hacer de la producción la base de la vida social, ha hecho de la conciencia humana de su propia creatividad, el objeto principal de su reflexión" (p. 68). 
En la hipermodernidad, "los seres humanos se enfrentan a su capacidad de crearse, transformarse, destruirse ellos mismos (...). Es, por lo tanto, en estas sociedades más creativas y autotransformadoras que la conciencia de sí mismo alcanza su mayor potencia, hasta el punto de hacer del respeto de esta creatividad el derecho más fundamental, el derecho al respeto de su dignidad" (p. 69). Ese derecho, cuyo contenido es más ético que cultural, tiene un carácter universal y profundamente democrático, sabiendo que "la democracia es el régimen político que sitúa a los derechos humanos fundamentales, que son la libertad, la igualdad y la dignidad de cada uno, por encima de todas las decisiones humanas y de las leyes" (p. 69). En otros términos, "los derechos humanos están por encima de las leyes" (p. 69).

En el segundo capítulo, dedicado a las etapas de la modernidad, el autor indica que su objetivo es "mostrar la necesidad, en todas las cosas, de eliminar las visiones materialistas, institucionales o idealistas de la historia y de sustituirlas por el análisis general de la modernidad" (p. 71). En efecto, "la interdependencia de los tres componentes de la modernidad nos conduce a reconocer que los actores del conflicto central en cada tipo de sociedad moderna comparten las mismas referencias a una civilización material y a una interpretación de la creatividad humana que reconoce la complementariedad, que es también conflictividad, entre dos fuerzas creadoras, una que proviene del actor dominante y otra del actor dominado" (p. 95).

En el caso del actor dominante, "la evolución conduce de la referencia a un principio superior (...) al llamamiento a un principio interno de creatividad, en particular al conocimiento racional cuyo rol es fundamental en las sociedades hipermodernas" (p. 95). El reconocimiento del rol de los actores dominados en el impulso dado a la creatividad es más difícil (p. 96). No en vano, es preciso notar "el reconocimiento cada vez más amplio de los derechos de cada individuo y, por consiguiente, de todas las categorías de ser considerados como fuentes de creación" (p. 96). Mientras que "las categorías dominantes adoptan, cada vez más, el punto de vista del sistema, (...) las categorías dominadas son, cada vez más, [deseosas] de afirmar unos derechos personales" (p. 96).

En las sociedades hipermodernas, "la creatividad humana está cada vez más enteramente y directamente cuestionada, haciendo aparecer [estas] sociedades como unos actores de la subjetivación [o], al contrario, como unas máquinas de desubjetivación" (p.98). No obstante, la subjetivación se extiende porque la creatividad "pone en movimiento a unos aspectos cada vez más diversos de la personalidad" (p.98).

En el tercer capítulo, dedicado al sujeto humano, Touraine observa que "los conflictos sociales no son unas expresiones de conflictos de intereses: son, ante todo, unos conflictos de interpretaciones. En cada debate y en cada [lucha] social, lo que 
está en cuestión es una interpretación social de una creatividad y de creaciones humanas que están cargadas de sentido cultural de la conciencia que las sociedades humanas tienen de su capacidad creadora, y que están así cargadas de valores universales" (pp. 114-115). Cuando los seres humanos son capaces de crear, transformar y destruir sus experiencias, el sentido que buscan y que se esfuerzan en alcanzar "se aproxima de ellos, de sus propias acciones y representaciones" (p. 115).

El hombre-sujeto de las sociedades hipermodernas "ha sustituido el más allá por la mirada interior de la conciencia y se desprende, cada vez más, de sí mismo como experiencia, porque desconfía de [la influencia] de las tradiciones, de las interpretaciones de sí mismo y de su historia” (p. 116). Pasa de la experiencia a la conciencia (p. 116). Cuando "el hombre moderno es creador, consciente de ser un sujeto, (...) se desprende de todas las experiencias vividas por la crítica, la melancolía, la duda y el descubrimiento del Otro" (p. 116). Cuando la conciencia de la creatividad humana es fuerte, no se arrima tanto a unas situaciones y a unos seres reales (p. 116). Y solo se pueden proteger y entender los derechos fundamentales "manteniendo constantemente una mirada crítica sobre las normas y las prácticas impuestas por las costumbres y las instituciones" (p. 117).

El sujeto, nos dice el autor, "nace de la aparición, en una red de prácticas sociales, del reto más elevado posible en un cierto tipo de sociedad (...). Lo más importante, por todas partes, es la conciencia en el [seno del] propio actor de la ruptura entre la lógica habitual de las situaciones en las cuales debe intervenir y el reto al que debe aportar una respuesta creadora y liberadora” (p. 119).

En las sociedades hipermodernas, los actores se enfrentan a una paradoja: "todos tenemos conciencia de nuestras identidades, de nuestras pertenencias, de nuestras elecciones; pero tenemos también conciencia que nuestra vida individual y colectiva está cada vez más profundamente transformada por unos conocimientos, unas voluntades, unos objetivos nuevos" (p. 120). Pasamos de la política a la ética y a "unas negociaciones por la afirmación de los derechos fundamentales que reconocemos como el único principio de legitimación de las leyes y de la autoridad" (p. 121). De hecho, la defensa "de los derechos fundamentales es la forma extrema, es decir la más directa, de la subjetivación" (p. 122), sabiendo que "la experiencia directa de una situación y la construcción del modelo de sociedad que corresponde a las necesidades centrales de esta sociedad son inseparables" (p. 122).

En las sociedades hipermodernas, la conciencia entera y directa de la creatividad humana representa lo que está en juego en los conflictos (p. 124), de modo que se dibuja un conflicto entre dos bandos opuestos: "uno, el de los dirigentes y de todos aquellos que están seducidos por su lógica, que quieren establecer un vínculo lo más directo y estrecho posible entre el modelo cultural de esta sociedad y las demandas individuales más inmediatas; y [otro, en el cual] se encuentran 
todos aquellos que intentan tener en cuenta, en la evaluación de los resultados, las referencias más directas a la figura de la subjetivación que corresponde, [en mayor medida], a la situación histórica concernida" (p. 124).

Touraine recuerda que, en su historia de la sexualidad, Michel Foucault "da vida a una historia cultural del sujeto" (p. 128). Pone de manifiesto "una nueva subjetivación, la cultura del Yo" (p. 128). En ese sentido, Foucault fue el primer explorador de una subjetivación con la suficiente exigencia (p. 128), siendo consciente de que la historia del sujeto está llena "de rupturas y de vuelcos" (p. 129).

Para el sociólogo galo, el universo subjetivizado de las sociedades hipermodernas es, a la vez:

- "Un universo de necesidades, de deseo sexual, de participación a redes de comunicación y de adquisición de bienes materiales, pasando por todos los consumos suscitados y amplificados por los mass media y las propagandas estatales" (p. 133).

- "Un universo de comunidades cuya fuerza está ilustrada en Europa por la violencia del rechazo de los refugiados (...) y por la importancia de los populismos xenófobos" (p. 133).

- "Un universo de ataques contra las concepciones del sujeto, contra todas las interpretaciones de la creatividad humana en nombre de una renaturalización del ser humano" (p. 133).

Esta ola de desubjetivación "busca destruir los logros de los pensamientos y de las acciones colectivas del periodo moderno por rechazo e incapacidad a entrar en la sociedad hipermoderna" (p. 134).

En esta última, nos dice el autor, estamos alejados "del triunfo de los determinismos tecnológicos y económicos. Esta nueva etapa de la modernidad está dominada, al contrario, por el enfrentamiento cada vez más directo del sujeto y del anti-sujeto, de la subjetivación y de la desubjetivación" (p. 138). En un mundo de comunicación social, de globalización económica y de poder total, "no se debe buscar defender las condiciones particulares de existencia y de acción del sujeto humano; son, al contrario, unos objetivos universales que [conviene] proponer a nuestra voluntad de subjetivación" (p. 139). De hecho, las sociedades hipermodernas se desarrollan, cada vez más rápidamente, en dos direcciones opuestas. "Por un lado, el incremento masivo de informaciones de las que disponemos sobre los comportamientos humanos, sobre nuestros consumos, [etc.]. Por otro lado, nos comportamos, cada vez más, como sujetos que poseen derechos y producen jurisprudencias, $y$, sobre todo, que buscan nuevas orientaciones culturales y nuevas fronteras entre lo antiguo y lo nuevo" (p. 141). 
Para Touraine, la primera respuesta que debemos aportar a la crisis intensa que atraviesa la modernidad, "desde el nacimiento de las revoluciones totalitarias, las crisis económicas mundiales y el auge de las políticas antidemocráticas, es aceptar o rechazar el porvenir cuya base material ya presente está formada por tres grandes elementos: la importancia de las comunicaciones, la globalización y el carácter total del poder" (p. 144). La segunda respuesta es que el porvenir no está determinado por las nuevas "tecnologías, la organización de la economía mundial y la nueva naturaleza (...) del poder (p. 145).

En el cuarto capítulo, que se interesa por la subjetivación y la desubjetivación, el sociólogo galo recuerda que "la subjetivación es la identificación de un individuo o de un grupo a la figura del sujeto que corresponde a su civilización y a sus prácticas" (p. 149). El proceso de subjetivación "es la toma de conciencia por un individuo o un grupo de sus derechos fundamentales, es decir universales del sujeto", que se pueden resumir por "libertad, igualdad, dignidad" (p. 149). La subjetivación "hace de un individuo o de un grupo los portadores de derechos humanos fundamentales, es decir los representantes de un movimiento social o, [mejor dicho], de un movimiento ético y democrático en la sociedad hipermoderna" (pp. 149-150).

Para el autor, la sociedad hipermoderna "no debe concebirse como una sociedad de técnicas, de intercambios y de comunicaciones, [o] como una sociedad de objetos, sino como una sociedad de sujetos humanos" (p. 151). Hoy en día, "surgen nuevas formas de liberación, en particular a través del conocimiento del mundo y, más aún, a través de la conciencia de sí mismo. Pero, esta hipermodernidad está constantemente y cada vez más amenazada por la creación de nuevos imperios y de nuevos comunitarismos identitarios" (p. 151). Ante ello, los derechos humanos fundamentales "deben ser transformados en fuerza personal y colectiva de acción, $\mathrm{y}$, por lo tanto, en instituciones de un nuevo tipo cuyo fin debe ser doble: liberar y reforzar la subjetivación, y luchar contra las formas y las fuerzas de desubjetivación" (pp. 152-153).

El sociólogo galo identifica una serie de misiones:

- "La misión de la vida debe poner en movimiento el conocimiento y los equipamientos necesarios para comprender y salvar la vida frente a las grandes enfermedades y frente a todas las formas de deterioro del cuerpo" (p. 153).

- "La misión del trabajo debe recordarnos que la creación es trabajo, llamamiento al conocimiento, invento y apertura de varios mercados, y descubrimiento de nuevos recursos" (p. 154).

- "La misión del porvenir debe abrir la sociedad a los jóvenes que están excluidos, en particular poniendo de nuevo en marcha el ascensor social, es decir luchando contra los efectos negativos del sistema educativo" (p. 155). 
- "La misión de acogida debe ser una prioridad en los países que, después de haber sido durante un largo periodo países de inmigración, (...) se cierran a los inmigrantes y a los refugiados" (p.155).

- "La misión de las edades de la vida (...) parece haber sido abandonada o haberse reducida a interminables discusiones sobre la edad de jubilación o el equilibrio [financiero] de la Seguridad social"' (p. 155).

Pero, no es suficiente con "hacer penetrar los derechos del sujeto en todos los aspectos de la vida social" (p. 156), ya que es importante reconocer la importancia de la "desinstitucionalización del sujeto" (p. 156).

En el quinto y último capítulo de esta primera parte, que se centra en los antiguos y nuevos movimientos sociales, Touraine subraya que "necesitamos una estrategia para despertarnos y convertirnos de nuevo en [actores], una estrategia que marcaría una ruptura más profunda con la tecnología política que hemos elaborado a lo largo de los siglos pasados" (p. 186). La primera novedad que observamos es "la sustitución, como marco privilegiado de acción, de las unidades de vida profesional por los territorios, por unos conjuntos definidos (...) como unos lugares de convivencia colectiva o de capacidad de acción, e incluso de voluntad de resistir y de sobrevivir" (p. 186). De hecho, "un territorio no es un recurso material [y] geográfico, una reserva de trabajo y de empleo, es, ante todo, una conciencia" (p. 187). En ese sentido, debemos pensar en términos de "cuenca de acción", "conciencia de país", etc. (p. 187).

Las personas están cada vez menos motivadas por una causa, un objetivo o unos valores, sino que lo están, de manera creciente, por la conciencia de su responsabilidad. Hoy en día, "un gran número de individuos se sienten responsables ante sí mismos [y] ante su imagen (...) como sujetos humanos" (p. 187). La conciencia de nosotros mismos como sujetos "se ha convertido en nuestro principal recurso" (p. 187). Por lo cual, "el objetivo principal de la política es crear y fomentar la subjetivación, es decir un cierto tipo de relación de los individuos y de los actores con sí mismos, una cierta toma de conciencia de [la] responsabilidad de estos actores hacia sí mismos como sujetos humanos" (pp. 187-188). Debe encarnarse en nuevos movimientos sociales que impulsan protestas éticas y democráticas, y enfrentarse a los anti movimientos sociales que "suscitan el escándalo, el horror y la condena moral" (p. 195).

En suma, "la primera y mayor fuerza resistente a todas las formas de poder total ha venido de la defensa del sujeto humano y de los derechos humanos fundamentales. El llamamiento al sujeto y, más concretamente, a la subjetivación y, [como] corolario, a la lucha contra la desubjetivación, es ante todo el rechazo de cualquier principio de unidad y de integración en el pensamiento y la acción de las sociedades modernas” (p. 206). 
En la segunda parte, titulada "La sociedad hipermoderna", el autor indica que esta sociedad se caracteriza por la conversión de la experiencia en conciencia. "Es de la conciencia de nosotros mismos, es decir de nuestra reflexión sobre nosotros mismos, sobre nuestro cuerpo, nuestra palabra, nuestra experiencia, y la interpretación que [hacemos] de ellos, que proviene el sentido de nuestras acciones [y] de toda nuestra experiencia" (pp. 215-216).

En el primer capítulo, dedicado a la civilización hipermoderna, Touraine subraya que la sociedad hipermoderna lleva a su extremo "el rol de la creatividad humana, hasta el punto de que ésta no sea [percibida] solamente como la interpretación de una civilización material sino que se convierte en el significado más general de la sociedad [en su conjunto]"' (p. 219). En la medida en que se basa en el logos, se impone por su propia naturaleza (p. 219). En su seno, "las grandes transformaciones económicas [provocan] la formación de nuevas élites dirigentes y, por lo tanto, de nuevas formas de planificación social” (p. 220).

En esta nueva sociedad, a la vez rápida y masiva, el fundamento de la acción se encuentra en su propia creatividad (p. 221). Es la razón por la cual, "esta sociedad hipermoderna tiene una conciencia entera y directa de su propia creatividad" ( $p$. 222). Y, aunque las nociones de clase y de conflictos de clase no se hallen en el centro del análisis, este tipo de sociedad está atravesado por el conflicto social (p. 222). El conflicto central en su seno es "cultural o ético y, por lo tanto, democrático" (p. 224). Así, "es en torno al estatus social y jurídico de los extranjeros que se han formado los conflictos sociales más intensos y que la [peor] expresión del Mal [es] el racismo y todas las prácticas que pretenden cerrar el acceso a una comunidad étnica, religiosa, lingüística e incluso geográfica" (p. 225). En la sociedad hipermoderna, "la oposición más profunda no es la que opone los ricos y los pobres, los amos y los esclavos; es la que opone las posesiones que se acumulan, las desigualdades de bienestar, de riqueza y de consumo, y (...) el sentido de la vida y de la acción tal como lo desarrolla la subjetivación" (pp. 227-228).

No en vano, si esta nueva civilización se expande a gran velocidad, "cada sociedad está marcada, no solamente por su grado de avance en la nueva cultura material y moral, sino también por el efecto de tres grandes formas de diferenciación: 1) su modelo de modernización la marca tan profundamente como su nivel de modernidad; 2) los juicios sobre los responsables políticos dependen, cada vez más, de su personalidad; y 3 ) las principales resistencias a unas decisiones políticas provienen (...) de las minorías más fuertemente integradas” (p. 233).

En el segundo capítulo, que se interesa por el poder total, el sociólogo galo indica que "la aspiración a reconocer un rol creativo a las emociones y a los sentimientos, tanto como al pensamiento racional, (...) es una tendencia profunda de la sociedad hipermoderna” (p. 241). Es “en la experiencia concreta más general, 
la de la relación entre natura y cultura, y, en particular, entre categorías de sexo, edad y $(. .$.$) origen, que [se articulan] los retos culturales en torno a los cuales$ se organizan los principales conflictos" (p. 242). Es preciso tener en cuenta al respecto que existe un vínculo directo "entre la sociedad de comunicación y los tipos de relaciones y de conflictos colectivos y personales que se desarrollan en la hipermodernidad" (p. 242), sabiendo que ésta se ve afectada por la globalización que provoca "una pérdida de importancia del control político sobre la vida individual y colectiva" (p. 242).

En la sociedad hipermoderna, "todos los actores, emisores y receptores de mensajes, están llamados a comunicar, es decir a movilizar todos los aspectos de su personalidad para interactuar con aquellos a los que se dirige" (p.243). Según Touraine, en ese tipo de sociedad, los poderes se ejercen en los ámbitos políticos, económicos y culturales, y, en particular, "los poderes político y económico se extienden espontáneamente (...) a los comportamientos y a las representaciones de los actores sociales" (p. 243). Los Estados que ejercen un poder total se convierten en imperios (p. 243). De hecho, "el poder de los nuevos imperios se basa, en gran parte, en la influencia ideológica, cultural y religiosa que ejercen, sea cual sea su tamaño" (p. 246).

Estas mutaciones tienen, “en los países occidentales, unas consecuencias más graves para la izquierda que para la derecha, porque la derecha tiene siempre un discurso más próximo a sus intereses, mientras que la izquierda se define más por unos proyectos y unos principios" (p. 247). La extensión de los poderes totales "no es solamente la consecuencia lógica del debilitamiento de los Estados nacionales y de la conquista de las mentes del mundo entero por las élites de poder (...); es también la consecuencia de la difícil formación de nuevos movimientos sociales y de nuevas políticas que les conferían una verdadera influencia allá donde las instituciones lo permiten" (p. 249). En ese panorama, los "nuevos movimientos sociales deben definirse en términos mundiales, pero siempre [a través de] unos objetivos y unas luchas ante todo éticos y democráticos" (p. 250).

En el tercer capítulo, que se interesa por los campos de subjetivación necesarios, Touraine indica que, "para que la voluntad de ser y de actuar como sujeto esté presente, [es preciso] que el posible actor, individual o colectivo, se sienta responsable y [capacitado para] actuar. Es la primera etapa de lo que se puede denominar 'la subjetivación de sí mismo'. Porque no es la acción la que crea el actor, sino el actor el que crea la acción” (p. 251).

Así, para el sociólogo galo, la liberación de las mujeres no puede limitarse a la igualdad de oportunidades, sino que debe traducirse en la "construcción de sí mismo como sujeto humano plenamente y directamente" (p. 259). De hecho, "ni los hombres ni las mujeres pueden realizar plenamente su propia subjetivación 
sin acabar con la dominación masculina y sin comprometerse en la vía de la autosubjetivación" (p. 259). A ese respecto, nos dice el autor, conviene "manifestar nuestra voluntad de subjetivación en el conjunto de las conductas, del deseo a la conciencia (...) de nuestra existencia como sujeto humano" (p. 260). Pero, es preciso, simultáneamente, "no dejarnos llevar hacia la reducción de la subjetivación a un simple bienestar asociado a una buena integración social” (p. 260).

Si Touraine concede una gran importancia, a la vez teóricamente y políticamente, al tema de la acogida de los refugiados, es porque "el conflicto central en la sociedad hipermoderna opone el universalismo de los derechos humanos a la defensa de las comunidades (...) particulares" (p. 261). De manera general, en ese tipo de sociedad, "el rol predominante de las comunicaciones conduce a la creación de poderes totales que pueden ser tanto de los mercados financieros como de los regímenes dictatoriales o de los anti movimientos sociales comunitaristas" (p. 262). Además, es preciso convencerse de que "la única respuesta eficaz al triunfo de los poderes totales que [alimentan] la amenaza, es la creación, la animación y el refuerzo rápido de nuevos movimientos sociales y de nuevas fuerzas políticas que asumirán (...) las razones de actuar de los nuevos movimientos en cuestión” (p. 264). Por último, es necesario prevenir las catástrofes ecológicas porque generarán "desplazamientos masivos de población, [cambios] no menos nocivos en la actividad económica” (p. 263).

En la tercera parte, titulada "de la sociedad industrial a la sociedad hipermoderna", cuyo primer capítulo analiza el binomio "modernizaciones y revoluciones", el pensador galo distingue tres tipos principales de modernización: "la modernización endógena, producida por los recursos y los actores de la sociedad estudiada, sobre todo cuando es dominante; la modernización dependiente, impuesta por la dominación de una potencia extranjera; [y] la modernización exógena que corresponde a los efectos inducidos por una sociedad sobre la modernización de otra que no domina políticamente y económicamente, pero sobre la cual ejerce una fuerte influencia”, (p. 271).

A partir de esta tipología, el autor insiste en la distinción entre movimiento social y acción revolucionaria, puesto que, "mientras que los movimientos sociales se refuerzan y se extienden en democracia, la acción revolucionaria prepara a menudo la victoria de un nuevo poder autoritario que justificará [el uso de la] violencia" (p. 279). De hecho, "las tres grandes revoluciones han preparado y nutrido unos regímenes autoritarios y jamás unas democracias" (p. 279). Asimismo, distingue los actores sociales centrales y los actores políticos periféricos, considerando que la sustitución de los primeros por los segundos es "un signo muy importante de declive histórico de un tipo de sociedad y de civilización" (p. 290). La proliferación de fuerzas populistas es prueba de ello (p. 290). 
En el último capítulo, dedicado a "los actores de la sociedad hipermoderna", Touraine incide en la complejidad creciente de esta nueva sociedad en razón de "la interdependencia creciente de los problemas socioeconómicos, de los [conflictos] internacionales y de [las dificultades] de la vida individual e interindividual" (p. 308). Además, las orientaciones culturales y los conflictos sociales que atraviesan las sociedades hipermodernas han sido transformados "por el paso de una economía de fabricación a una economía de comunicación, es decir de la acción sobre la naturaleza a la acción sobre los seres humanos por la modificación de sus conductas, pero también por el efecto (...) de la educación” (p. 310). A todo ello, es preciso añadir otras dos transformaciones: por una parte, "la globalización (...) definida en términos más políticos que económicos" (p. 310), y, por otra parte, "la desocialización de ciertas conductas [asociadas] a las minorías sexuales" (p. 312).

Según el autor, "nuestra capacidad a construir una nueva modernidad depende, en primer lugar, de nuestra capacidad a comprometernos en la vía de la modernización" (p. 320). El principal obstáculo al que se enfrentan las sociedades contemporáneas es "el capitalismo financiero, en la medida en que [orienta] una gran parte del capital en dirección de actividades no productivas en nombre de la búsqueda cada vez más excesiva de los beneficios” (p. 325). Para superarlo, es preciso que, "de los dirigentes a los ciudadanos portadores de proyectos, cada uno [adquiera] cierta confianza en un proyecto general susceptible de acoger su propio proyecto en el seno del pacto (...) democrático" (p. 328). Es necesario, asimismo, que la política aspire a "la integración más masiva posible de la población en la solidaridad (...), una población dotada de derechos fundamentales más completos posibles y [que padezca] exclusiones muy limitadas" (p. 328). En los tres ámbitos principales de la innovación social, que son la investigación, la educación y la sanidad pública, "se trata de transformar unas administraciones en instrumentos de una participación social claramente afirmada como un objetivo [fundamental]" (p. 328).

Al término de la lectura de Défense de la modernité, es preciso reconocer la aportación realizada por el autor, de libro en libro, a la elaboración de una sociología del sujeto y de la hipermodernidad. Gracias a su amplia cultura en humanidades, que bebe especialmente de la filosofía y de la sociología, con abundantes referencias a la literatura y a las artes, profundiza los conceptos de sujeto humano, subjetivación, desubjetivación y nuevos movimientos sociales, en una visión coherente y sistematizada. Su estilo fluido y su lenguaje asequible convierten la lectura del presente libro en un placer.

No en vano, se trata más de un ensayo filosófico que de una investigación sociológica y se caracteriza por un alto nivel de abstracción y de generalización. De hecho, Touraine cae en ciertas generalidades y simplificaciones, especialmente 
cuando aborda determinados enfoques y debates, como el determinismo económico. Además, al volver uno y otra vez sobre los mismos conceptos, la obra aporta pocas novedades con respecto a libros anteriores, tales como La fin des sociétés (2013) y Nous, sujets humains (2015). Asimismo, se observan notables redundancias que dan una sensación de repetición. A su vez, esta obra, como las anteriores, se caracteriza por una fuerte dimensión normativa, en la medida en que Touraine insiste sobre la sociedad tal y como debería ser o la manera en que los actores deberían actuar frente a los principales desafíos a los que se enfrenten. Por último, su exposición de los tipos de sociedad correspondientes a periodos históricos diferentes cae a menudo en el evolucionismo.

En cualquier caso, la lectura de esta obra, que aparece como un adiós personal y un testamento intelectual del autor, es recomendable para profundizar nuestra comprensión de las sociedades hipermodernas.

BIBLIOGRAFÍA

Touraine, A. (2013): La fin des sociétés. París: Seuil.

Touraine, A. (2015): Nous, sujets humains. París: Seuil.

Touraine, A. (2018): Défense de la modernité. París: Seuil. 